\title{
Adopción de Herramientas Educativas en Familia y Escuela.
}

\author{
González Bohórquez, Myriam Yohana \\ Corporación Universitaria Minuto de Dios, Zipaquirá, Colombia. \\ myriam.gonzalez@uniminuto.edu \\ twomay84_9@hotmail.com
}

\section{Resumen}

Actualmente la sociedad pasa por un desequilibrio social donde no se conoce la incidencia de problemáticas que afectan continuamente a un individuo, por lo cual se ha visto la necesidad de aborda el entorno familiar como núcleo de la sociedad y el entorno escolar como acompañamiento continuo a la formación que precede de la familia. Es así como surge la propuesta de retomar diferentes tipos de familia para delimitar problemáticas que se presentan, y con ello diseñar actividades didácticas que permitirán generar espacios de enseñanza- aprendizaje, consolidándose así material pedagógico que permitirá realizar una evaluación y seguimiento del aprendizaje en cada entorno familiar y escolar. Los resultados que se evidencien de la aplicación e interacción con el material pedagógico, se retomaran para consolidación de rutas de atención prevención que utiliza el Trabajador Social para manejar y mitigar problemáticas que se evidencian actualmente.

Es necesario que hoy en día se genere espacios para formar a la familia en contextos diversos que no permiten la estabilidad de una sociedad, por cual fue indispensable comparar y asociar procesos de enseñanza- aprendizaje en el entorno familiar y escolar, siendo estos los dos lentes sociales de formación de un individuo. La relación de los mismos consolidará información que muestra la tipología familiar, contexto, estructura organizativa, acciones que sigue cada núcleo familiar para el condicionamiento de un modelo educativo que determine criterios, particularidades de la interacción y convivencia familiar para evidenciarse en su entorno próximo.

\section{Abstract}

Currently the society is going through a social imbalance where the incidence of problems that continuously affect an individual is not known, which is why we have seen the need to address the family environment as the core of society and the school environment as a continuous accompaniment to the previous training of the family. This is how the proposal to return to different types of family to define problems that arise, and thus design teaching activities that will generate teaching-learning spaces, consolidating pedagogical material that will allow an evaluation and monitoring of learning in each environment family and school. The results that are evidenced by the application and interaction with the pedagogical material, will be retaken for the consolidation of prevention care routes used by the Social Worker to manage and mitigate problems that are currently evident. It is necessary that nowadays spaces are generated to train the family in diverse contexts that do not allow the stability of a society, for which it was indispensable to compare and associate teaching-learning processes in the family and school environment, these being the two lenses social training of an individual. Their relationship will consolidate information that shows the family typology, context, organizational structure, actions followed by each family nucleus for the conditioning of an educational model that determines criteria, particularities of interaction and family coexistence to be evident in their immediate environment.

Palabras clave: Familia, Enseñanza-aprendizaje y Educación Comparada.

Keywords: Family, Teaching-learning and Comparative Education.

\section{INTRODUCCIÓN}

La investigación se consolida en análisis del entorno familiar y escolar como medios de acción que evidencian vacíos de aprendizaje permanente, ya sea por no tener una directriz específica de enseñanza o por privilegiar escenarios que evidencias crecimiento laboral o profesional, antes que el familiar y escolar. Se requiere entonces de ver entorno familiar como un sistema social, que está conformada por tres subsistemas básicos: fraterno, conyugal, parentesco - filial, que a su vez están interrelacionados relacionados con el suprasistema (el vecindario, la comunidad, el trabajo, la escuela y la escuela como un todo); (...) «Es la única sociedad vinculada con los demás sistemas. Lo que ocurre con un miembro repercute en los demás sistemas, mediante un intercambio simbiótico de energía, 
información, cultura, normas y funciones» (Quintero, 2007, p 59). Por ello se requiere de observar, indagar, contrastar y comparar el entorno familiar de diferentes tipos de familia para consolidar información que permita crear herramientas pedagógicas que logren direccionar un aprendizaje guiado para fortalecer estructura familiar que se evidencia frágil por los cambios sociales que surgen de una globalización desenfrenada.

La familia es el primer lente social que permite identificar y asociar comportamientos vistos a nivel social, es de anotar que este entorno muestra un grupo de convivencia basado en el parentesco, la filiación y la alianza; sus miembros están ligados por sangre o por afinidad, lo cual crea una serie de relaciones, obligaciones y emociones. Si entonces el espacio para la socialización del individuo, el desarrollo del afecto y la satisfacción de necesidades sexuales, sociales, emocionales y económicas, y el primer agente transmisor de normas, valores, símbolos, ideologías e identidad, donde se focalizan las acciones de las instituciones (Quintero, 2007.p 59).

Entonces la familia concibe al individuo como agente social logrando evidenciar las bases para convivir en sociedad, dando paso a un proceso de formación que acompaña e integra lo aprendido en familia, siendo entonces el entorno escolar el siguiente lente para estudio y evidencia de lo que es el aprender a aprender. Para ello es necesario incursionar en los sistemas educativos globales que permitan dilucidar el actuar y el accionar de la educación en un contexto local, con ello se establecerán estudios que logran mostrar y diferencias los métodos de enseñanza para enriquecer cada modelo de acción pedagógico.

Cuando se habla de enseñanza- aprendizaje se direcciona a establecer características dadas de acuerdo al manejo que el docente desempeña en el aula de clases, determinando que cada proceso educativo evidencia diversos mecanismos que se pueden compactar y generar un método de enseñanza que permita al educando obtener conocimiento global de manera local. Entonces la educación se consolida como proceso de adquisición de conocimiento y un escenario para evidenciar la esencia de un sujeto que se involucra en un entorno diverso y abierto a comparación de su entorno familiar.

Por ello cuando se determina una educación comparada tiene como objeto de estudio la realidad o parte de ella, dentro de cada área académica, observándose la comparación de los hechos educativos y de las relaciones que le unen a su medio (García, 2012), mostrando la educación comparada como un instrumento imprescindible para la elaboración y ejecución de reformas e innovaciones educativas ( García, 2012), que se requieren para la implementación de modelos educativos que permitan argumentar, interpretar y proponer sobre la realidad social actual.

\section{ENTORNO FAMILIAR}

Desde la perspectiva de Minunchin define «La familia es un grupo de personas, unidas emocionalmente y/o por lazos de sangre, que han vivido juntos el tiempo suficiente como para haber desarrollado patrones de interacción e historias que justifican y explican tales patrones». (1998, pág. 12); por lo cual en el contexto familiar se desarrollan habilidades de interacción, construyendo poco a poco una historia, evidenciándose la familia como primera instancia en el desarrollo escolar del niño, pues es allí donde van formando valores, actitudes, posturas que más tarde afianza y demuestra en su diario vivir.

El grupo familiar como una constitución que se considera la más importante en la vida del ser humano puesto que en su desarrollo integral tienes muchas necesidades biológicas (alimentación, sueño, descanso abrigo a la reproducción etc), como necesidades que se refieren a áreas afectivas y sociales. (MORA, 1986, p.1). Es de anotar que cada integrante de la familia goza de capacidades intelectuales únicas, con talentos que son poco aprovechados, sometiéndose en un círculo vicioso tradicional, donde cada miembro da respuesta a particularidades que surgen de conflictos interpersonales, visibilizándose en disputas familiares que muestran disfuncionalidad social.

Los integrantes de cada entorno familiar dan respuesta a largos periodos de negociación, compromiso, reorganización y competición. (Minunchin, 1998, pág. 12), este proceso de intercambio permite mostrar factores que influyen para establecer normas, roles, responsabilidades y acciones dependiendo del contexto o situación de la dinámica familiar. Es necesario reconocer cada aspecto familiar para condicionar un modelo de enseñanza-aprendizaje que muestre lineamientos familiares que demuestren la convivencia, el manejo y la integralidad familiar. 
A lo largo de la historia ha sido reconocida como la institución social primordial para el desarrollo social, por ello se hizo necesario la identificación del estado actual de la familia en cuanto a nivel estructural, funcional y vital al igual que sus características socio-demográficas, socio-culturales, socio- económico y psicológico donde las pioneras en este proceso fueron las ONGS y universidades tanto públicas como privadas quienes enfatizaron la investigación hacia las conductas derivadas de la relación socio- familiar que tienen o llevan a diario (Quintero, 2001)

\subsection{Enseñanza- Aprendizaje}

Los ambientes de aprendizaje pueden ser variados, más aún, cuando se maneja la diversidad social y la proyección a un desarrollo centralizado que permita la cooperación para el desarrollo aglutinado en un conjunto de políticas, actividades, proyectos y programas elaborados para colaborar con personas, pueblos, regiones o países que precisan de apoyos para mejorar sus condiciones de vida, para desarrollar plenamente sus potencialidades hasta alcanzar una vida digna y autónoma (Celorio, G. 2007). Por esto se requiere de un conocimiento previo del ambiente que se pretende evaluar, ya que su aplicabilidad puede presentar variaciones evidentes que demuestren la tipología familiar, su dinámica, el manejo del sujeto frente al lente familiar y escolar.

Partiendo de lo anterior se puede anotar que es fundamental la utilización de todos los elementos que integran el proceso de formación de la persona, y que la libertad sin dirección es vacía. Para ello el mundo de la educación , por sus diversos agentes y todos los niveles de intervención debe ocuparse deliberadamente de identificar un modelo de educación continuo que pretenda moldear e instaurar mecanismos de participación activa, desarrollando un compromiso, de ayuda y de preocupación por las otras personas, aun a pesar de las diferencias en los niveles de habilidades, las diferencias de raza de género y de clase social, a través de estrategias competentes a nivel social y actitudes prosociales (Chaux, E. 2013).

\subsubsection{Educación Comparada}

La educación comparada tiene como objeto de estudio la realidad o parte de ella, dentro de cada área académica, observándose la comparación de los hechos educativos y de las relaciones que le unen a su medio (García, 2012), mostrando la educación comparada como un instrumento imprescindible para la elaboración y ejecución de reformas e innovaciones educativas ( García, 2012), que se requieren para la implementación de modelos educativos que permitan argumentar, interpretar y proponer sobre la realidad social actual.

Entonces la Educación Comparada se caracteriza por el estudio de los problemas educativos con un enfoque multi y transdisciplinario, que sigue una vocación internacional y multicultural, examinando aspectos como la relación entre la educación y el desarrollo económico, político y social (López, 2008). Es por ello que se revisa las estrategias de enseñanza y métodos de aprendizaje que instituciones colombianas aplican para el direccionamiento y manejo convivencial en sus estudiantes, permitiendo observar la interacción en el ente familiar y escolar. Al verificar las necesidades familiares y escolares que el niño requiere, se establecerán mecanismos de acción que condicionan al sujeto a recibir información acertada para fortalecer un crecimiento emocional y físico adecuado que propenda un mejor calidad de vida.

\section{DISCUSIÓN Y CONCLUSIONES}

Hoy es necesario retomar estrategias educativas que permitan conocer y distinguir cada entorno familiar, dado que cuando se toma la tarea de ser padres no se está preparado y la práctica es la que permite adquirir conocimiento. Esto permite dar paso a la gran incógnita de cómo elaborar una herramienta didáctica que permita ser la guía para que un padre afronte situaciones familiares que pueden desestabilizar o incidir en la estabilidad emocional y social de un sujeto que se desenvuelve en escenarios diversos. 
Cada proceso realizado durante el proyecto social se fundamentará en el análisis y comparación de estrategias de enseñanza y métodos de aprendizaje que condicionen al sujeto a recibir información oportuna para fortalecer su capacidad cognitiva y emocional al momento de interactuar con circunstancias que no permiten la estabilidad de su entorno próximo.

La creación de ambientes de aprendizaje condicionan a fortalecer lazos familiares y escolares que motiven al sujeto a valorar y desempeñarse como persona única, que logre desarrollar mecanismos de resolución de conflictos frente a circunstancias ocasionales y frecuentes.

\section{REFERENCIAS BIBLIOGRÁFICAS}

Celorio, G. (2007). «Educación para el desarrollo». En: Celorio, G. \& López. A. (Coords.). Diccionario de Educación para el Desarrollo. Bilbao: Hegoa. Recuperado de: http://pdf.hegoa.efaber.net/entry/content/158/diccionario_2.pdf.

Chaux, E. (2013). Competencias ciudadanas: de los estándares al aula. Una propuesta de integración en las áreas académicas. Universidad de los Andes. Bogotá.

García, J. L. (2012). Naturaleza y límites de la educación comparada. En J. L. García, M. J. García \& E. Gavari (coordinadores), La educación comparada en tiempos de globalización (pp. 15-42). Madrid, España: Universidad Nacional de Educación a Distancia (UNED).

López, A. (2008). Retos metodológicos de la educación comparada en la sociedad global. Revista de Universidad y Sociedad del Conocimiento, 5(1), 1-9.

Mora Rojas, R. (1986) problemas de la familia y la niñez en costa rica, Universidad de Costa Rica.

Minunchin, S. (1998), El Arte de la terapia familiar, ediciones Paidós ibérica S.A Barcelona.

Quintero, A. (2007), Diccionario especializado en familia y género, 1 edición, Buenos Aires, Lumen. 